\title{
Erratum to: Acculturation and Depressive Symptoms in Hispanic Older Adults: Does Perceived Ethnic Density Moderate their Relationship?
}

\author{
Kyung Hwa Kwag' ${ }^{1}$ Yuri Jang ${ }^{2}$ David A. Chiriboga ${ }^{3}$
}

Published online: 31 March 2015

(c) Springer Science+Business Media New York 2015

Erratum to: J Immigrant Minority Health (2012)

\section{4:1107-1111}

DOI 10.1007/s10903-011-9569-z

There is an error in the Acknowledgement section.

The "Sacramento Area Latino Study on Aging" (SALSA) was incorrectly identified as being managed by the Institute on Aging (IOA) at the University of Wisconsin-Madison and incorrectly identified as being supported by the National Institute on Aging (NIA) grant P01AG020166, which instead funds the MIDUS study administered by IOA. Although SALSA may be funded by NIA, the grant number acknowledged is incorrect.

The online version of the original article can be found under doi:10.1007/s10903-011-9569-z.

Kyung Hwa Kwag

kkwag@slu.edu

1 Department of Sociology and Criminal Justice, College of Arts and Sciences, Saint Louis University, 3750 Lindell Blvd., St. Louis, MO 63108, USA

2 School of Aging Studies, University of South Florida, Tampa, FL, USA

3 Department of Child and Family Studies, University of South Florida, Tampa, FL, USA 